\title{
Lateral bone density measurements in osteoarthritis of the lumbar spine
}

\author{
D J Peacock, P Egger, P Taylor, M I D Cawley, C Cooper
}

\begin{abstract}
Objective-To investigate whether spinal osteoarthritis (OA) is responsible for the common finding that lumbar spine bone mineral density (BMD) is greater when measured in the anteroposterior plane than when measured in the lateral plane.

Methods-We studied lateral spine radiographs from 63 women who attended a hospital outpatient department for bone density measurement and who also underwent lumbar spine radiography. Osteoarthritis was assessed using both the Kellgren and Lawrence scale and a scoring system for osteophytosis. Bone density was measured in the anteroposterior and lateral planes using a Hologic QDR-2000 instrument.
\end{abstract}

Results-The mean anteroposterior BMD $\left(0.92 \mathrm{~g} / \mathrm{cm}^{2}\right)$ was significantly greater than the lateral BMD $\left(0.59 \mathrm{~g} / \mathrm{cm}^{2}\right)(p<0.01)$, and the difference between anteroposterior and lateral measurements was significantly associated with both increasing Kellgren and Lawrence score and osteophyte score, even after adjustment for age.

Conclusion-These data suggest that spinal $O A$ is a major cause of the difference between anteroposterior and lateral BMD and that lateral BMD may provide a more accurate representation of true vertebral body bone density in patients with OA of the lumbar spine.

(Ann Rheum Dis 1996; 55: 196-198)

Measurement of bone mineral density (BMD) is an important technique in both diagnosis and management of osteoporosis. Vertebral BMD is usually measured in the anteroposterior plane, though this method may give falsely high values in the presence of lumbar spondylosis or osteoarthritis, especially when associated with osteophytes. ${ }^{1-3}$ BMD measured in the lateral plane does not include the posterior process of the vertebral body or any coexisting osteophytes and consequently may give a more accurate representation of true vertebral body $B M D$. We investigated the effects of lumbar spine osteoarthritis on both anteroposterior and lateral BMD in a cohort of women attending an outpatient clinic for BMD measurement.

Patients and methods

Sixty three women (mean age $61 \cdot 2$ years, range 34-87 years), attending the Medical Physics
Department for BMD measurement, were studied. BMD was measured in both anteroposterior and lateral planes, at the same appointment, by dual energy $x$ ray absorptiometry (DEXA) using a Hologic QDR 2000 instrument. The reproducibility of BMD measurements in the anteroposterior and lateral projections has been shown to be $0.8 \%$ and $2 \cdot 1 \%$, respectively, using this instrument. All subjects had undergone standardised lumbar spine radiography within the preceding three years. Osteoarthritis was assessed by a single blinded observer using the Kellgren and Lawrence scale ${ }^{5}$ with a of score $0-4$ ( $4=$ maximum arthritis). In addition, osteophytosis was scored on a scale of 0-3 (3 = maximum osteophytosis) at each individual lumbar vertebra using the method of Lane et al. ${ }^{6}$ The presence or absence of facet joint disease was noted.

STATISTICAL ANALYSIS

The data were analysed using linear regression for continuously distributed variables, and analysis of variance to compare groups. Adjustments for age were performed in a multiple regression model.

\section{Results}

The mean anteroposterior BMD was significantly greater than the mean lateral BMD at L3 $\left(0.92 \mathrm{~g} / \mathrm{cm}^{2}\right.$ and $0.59 \mathrm{~g} / \mathrm{cm}^{2}$, respectively, $\mathrm{p}<0.001$ ) (fig 1). Each individual subject also had a greater anteroposterior BMD than lateral $\mathrm{BMD}$. Increasing anteroposterior BMD was associated with greater Kellgren and Lawrence scores $(p=0.002)$ and increasing osteophyte score $(p<0.001)$ when adjusted for age (table). No such association was found with lateral BMD measurements, even after adjustment for age as a confounder.

The percentage difference between anteroposterior and lateral $\mathrm{BMD}$ measurements ([(anteroposterior BMD-lateral BMD)/ anteroposterior $\mathrm{BMD}] \times 100$ ) was significantly associated with increasing Kellgren and Lawrence scores $(p=0.003$; analysis of variance) and increasing osteophyte score ( $p=0.004$; analysis of variance) (fig 2). Adjustment for age did little to alter these associations and they remained highly significant (Kellgren and Lawrence score: $p=0.008$; osteophyte score: $p=0.011$ (analysis of variance)). In addition, the associations were not related to the extent of facet joint osteoarthritis, as assessed from the anteroposterior spinal radiographs. 


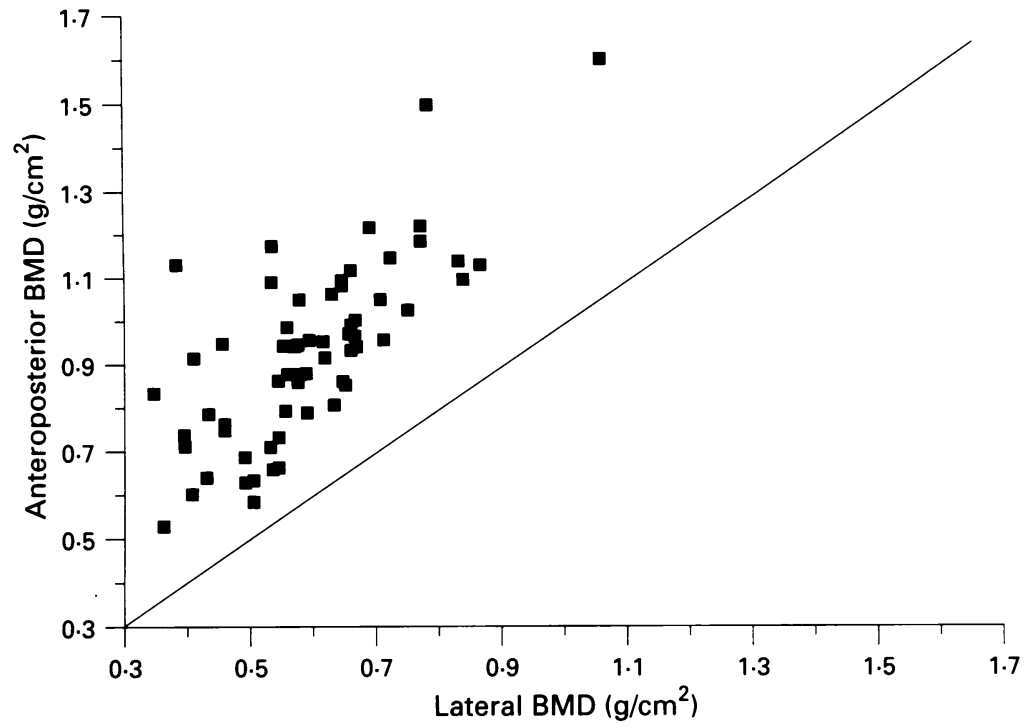

Figure 1 Relation between lumbar spine bone density values obtained in anteroposterior (mean $0.93(S D 0.21) \mathrm{g} / \mathrm{cm}^{2}$ ) and lateral (mean $0.59(0.13) \mathrm{g} / \mathrm{cm}^{2}$ ) projections among 63 women.
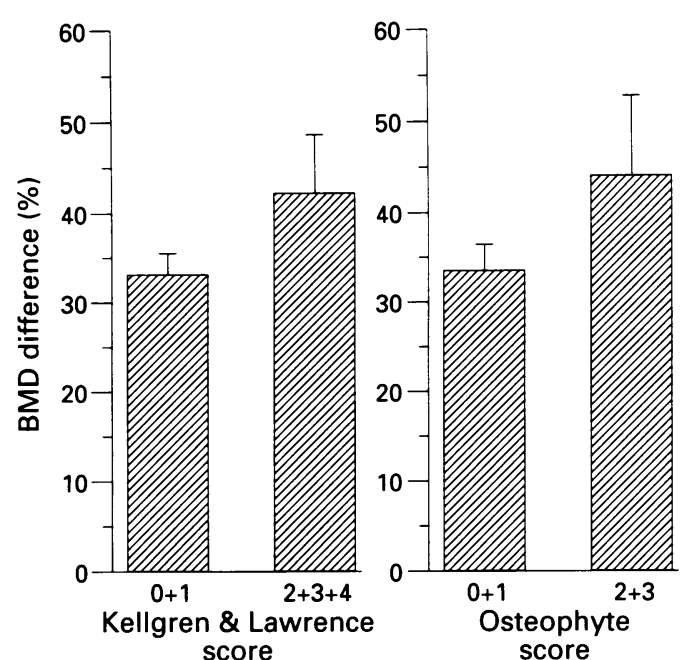

Figure 2 Difference (as percentage of the anteroposterior value) in lumbar spine bone density on anteroposterior and lateral projections among 63 women, categorised according to degree of osteoarthritis using an overall Kellgren and Lawrence score, and using a score for osteophyte alone (at L3).

Lateral DEXA has a similar accuracy, but considerably lower reproducibility, compared with anteroposterior DEXA. ${ }^{7}$ Moreover, one study has shown that BMD measured by lateral DEXA and BMD measured by quantitative computed tomography are more closely correlated than is either measure with anteroposterior BMD. ${ }^{8}$ The main difficulties associated with lateral DEXA are caused by the ribs and the pelvic rim interfering with $\mathrm{L} 2$ and $\mathrm{L} 4$ BMD, respectively. ${ }^{9}$ As a consequence of these problems, we chose L3 BMD measurement for this study. We also used both Kellgren and Lawrence and individual osteophyte scores, as the former is a global assessment of lumbar spine osteoarthritis, whilst the latter is an assessment of each individual vertebra. As expected, there was a significant positive correlation between the two methods. A further practical difficulty with the lateral measurement in arthritic patients was positioning of the arms.

There remains uncertainty over the comparative discriminatory ability of lateral and anteroposterior BMD measurements for vertebral deformity. Del Rio et al ${ }^{10}$ compared 185 patients with 1554 controls and found that the anteroposterior measurement discriminated between the two groups better than did the lateral one (mean anteroposterior difference $-3.0 \mathrm{SD}$; mean lateral difference $-2.5 \mathrm{SD}$ ). Similar findings were reported by Bjarnason et al. ${ }^{11}$ However, these observations contrast with those of a third study ${ }^{12}$ in which lateral measurements were found to discriminate spine fracture cases from controls more effectively than those made in the anteroposterior projection.

Several studies are consistent with a generalised increase in bone mineral density among patients with osteoarthritis. Although facet joint osteoarthritis was not a significant determinant of BMD in our study, it remains possible that the negative association between osteoarthritis and osteoporosis stems from a combination of real and artefactual changes. 
Aortic calcification is another potential confounder, but it was seen in only two patients in our group, probably because the mean age of the subjects was only about 60 years. Furthermore, the contribution of aortic calcification to anteroposterior BMD is controversial and a recent study suggested that it had little effect. ${ }^{13}$ Finally, this study may overestimate the importance of spinal OA. The subjects were selected on the basis that they had required recent spine radiography, and were thus likely to have had a greater prevalence of spine OA than that expected in the general population.

As age is associated with both a decrease in BMD and an increase in the prevalence of osteoarthritis, we expected that age adjustment might reduce the relationship between the anteroposterior-lateral difference and Kellgren and Lawrence grade. Although the prevalence of osteoarthritis increased with age (mean age of subjects with Kellgren and Lawrence scores 0 or $1=57.5$ years, with Kellgren and Lawrence scores $2-4=73.9$ years), we found only a weak positive relationship between anteroposteriorlateral difference and age $(r=0 \cdot 17, \mathrm{p}=0 \cdot 17)$. Furthermore, in the regression model which included both age and Kellgren and Lawrence grade, the latter exerted the greatest effect, with little additional variation in anteroposteriorlateral difference explained by age.

The lower reproducibility and technical difficulties associated with lateral BMD measurement clearly serve to limit the application of this method, but our results suggest that lateral rather than conventional anteroposterior BMD may be more accurate among subjects with degenerative disease of the lumbar spine. Longitudinal studies are required to explore further the diagnostic sen- sitivity of this approach in the general population.

This manuscript was prepared by Katy Cuninghame. We thank Vanessa Cox and Paul Winter for assistance with computing support.

1 Masud T, Langley S, Wiltshire P, Doyle D V, Spector T D. Effect of spinal osteophytosis on bone mineral density measurements in vertebral osteoporosis. $B M \mathcal{F} 1993 ; 307$ $172-3$.

2 Orwoll E S, Oviatt S K, Mann T. The impact of osteophytic and vascular calcification on vertebral bone mineral density measurements in men. $\mathfrak{f}$ Clin Endocrinol Metab density measurem 70 : 1202-7.

3 Dawson-Hughes B, Dallal G E. Effect of radiographic abnormalities on rate of bone loss from the spine. Calcif Tissue Int 1990; 46: 280-1.

4 Blake G M, Jagathesan T, Herd R J M, Fogelman I. Dual $\mathrm{X}$-ray absorptiometry of the lumbar spine: the precision of paired anteroposterior/lateral studies. Br $\mathcal{F}$ Radiol 1994; 67: $624-30$.

5 Kellgren J H, Lawrence J S. Radiological assessment of osteoarthritis. Ann Rheum Dis 1957; 16: 494-501.

6 Lane N E, Nevitt M C, Genant H K, Hochberg M C. Reliability of new indices of radiographic osteoarthritis of the hand and hip and lumbar disc degeneration. of Rheumatol 1993; 20: 1911-8.

7 Mazess R B, Gifford C A, Bisek J P, Barden H S, Hanson J A. DEXA measurement of spine density in the lateral projection. 1: Methodology. Calcif Tissue Int 1991; lateral projectic
49: $235-9$.

8 Finkelstein J S, Cleary R L, Butler J P, et al. A comparison of lateral versus anterior-posterior spine dual energy $x$-ray absorptiometry for the diagnosis of osteopenia. $f$ Clin Endocrinol Metab 1994; 78: 724-30.

9 Larnach T A, Boyd S J, Smart R C, Butler S P, Rohl P G, Diamond $T H$. Reproducibility of lateral spine scans using dual energy $x$-ray absorptiometry. Calcif Tissue Int 1992; 51: 255-8.

10 Del Rio L, Pons F, Juguet M, Setocrin F J, Setocrin J. Anteroposterior versus lateral bone mineral density of Anteroposterior versus lateral bone mineral density of spine assessed by dual

11 Bjarnason K, Nilas L, Hassager C, Christiansen C. Dual energy $\mathrm{X}$-ray absorptiometry of the spine-decubitus lateral versus anteroposterior projection in osteoporotic women: comparison to single energy $\mathrm{X}$-ray absorptiometry of the forearm. Bone 1995; 16: 255-60.

12 Jergas M, Breitenseher M, Gluer C C, Yu W, Genant J K Estimates of volumetric bone density from projectional measurements improve the discriminatory capability of dual X-ray absorptiometry. F Bone Miner Res 1995; 10 1101-10.

13 Drinka P J, Bauwens S F, De Smet A A. Lack of correlation between aortic calcification and bone density. Wis Med $\mathcal{Y}$ 1992; 91: 299-301. 